\title{
Erratum to: Synthesis of Fused Heterocyclic Systems Derived from 2-Aryl-1,2,3,4-Tetrahydroquinolines
}

\author{
M. N. Zemtsova ${ }^{a}$, Yu. A. Golovko ${ }^{a}$, Yu. A. Gruzd ${ }^{a}$, S. V. Kulemina ${ }^{a}$, \\ M. R. Baimuratov ${ }^{a, *}$, and Yu. N. Klimochkin ${ }^{a}$ \\ ${ }^{a}$ Samara State Technical University, Samara, 443100 Russia \\ *e-mail: baymuratovmr@yandex.ru \\ Submitted December 1, 2021; accepted for publication December 1, 2021
}

DOI: $10.1134 / \mathrm{S} 1547477122010113$

During our further research on gravity models with non-linear symmetry realization we found out that in our paper entitled "Inflationary Solutions in the Simplest Gravity Model with Conformal Symmetry" there was a small mistake. Fortunately it does not influence the general result.

So, the stress-energy tensor for the considered model leads to equations:

$$
\begin{gathered}
-3 \frac{\ddot{a}}{a}=5 k\left(4 f_{1} \dot{\psi}^{2}+f_{2} \dot{\sigma}^{2}+f_{3} \dot{\sigma}_{(\alpha)} \sigma^{(\alpha)}\right), \\
2 \dot{a}^{2}+a \ddot{a}=0
\end{gathered}
$$

except written in the paper after Eq (3). Therefore, the analysis becomes simpler, and the absence of inflation directly follows from (1):

$$
\ddot{a}=-2 \frac{\dot{a}^{2}}{a}<0 .
$$

Thus, our conclusion about the absence of inflation confirms.

The original article can be found online at https://doi.org/10.1134/S1070428021050055 